Relations industrielles

Industrial Relations

\title{
De l'entreprise libérale à la libre entreprise
}

\section{Marcel Clément}

Volume 4, numéro 2, octobre 1948

URI : https://id.erudit.org/iderudit/1023432ar

DOI : https://doi.org/10.7202/1023432ar

Aller au sommaire du numéro

Éditeur(s)

Département des relations industrielles de l'Université Laval

ISSN

0034-379X (imprimé)

1703-8138 (numérique)

Découvrir la revue

Citer cet article

Clément, M. (1948). De l'entreprise libérale à la libre entreprise. Relations

industrielles / Industrial Relations, 4(2), 11-14. https://doi.org/10.7202/1023432ar

Tous droits réservés @ C Département des relations industrielles de l’Université Laval, 1948
Ce document est protégé par la loi sur le droit d'auteur. L'utilisation des services d'Érudit (y compris la reproduction) est assujettie à sa politique d'utilisation que vous pouvez consulter en ligne.

https://apropos.erudit.org/fr/usagers/politique-dutilisation/ 


\section{Bulletin des nelations industrielles}

Volume 4, numéro 2

QUÉBEC

Octobre 1948

Publie par le

Département des relations industrielles, Faculté des sciences sociales, Université Laval.

Georges-Henri Lévesque, o.p., doyen GÉrARD TrEmblay, directeur GÉrARD Dion, sous-directeur Jean Gagné, secrétaire Charles Bélanger, administrateur

Le Bulletin paraît mensuellement de septembre à juin (dix numéros par année). Abonnement annuel: Canada: $\$ 1.50$; étranger: $\$ 2.00$. Vingt-cinq cents le numéro.

\section{Adressez toute correspondance au secrétaire de rédaction GÉrard Dion \\ 2, rue de l'Université, Ouébec.}

Bulletin des relations industrielles

\begin{tabular}{llll}
\hline Volume 4, numéro 2 & octobre 1948 \\
\hline
\end{tabular}

Sommaire

De l'entreprise libérale d

la libre entreprise

Marcel Clément.

Un directeur du personnel S.V.P.

Gérard Tremblay

Les sciences sociales et l'homme

Hon. Antonio Barrette. .. . .

L'Université Laval au

service du peuple.

Les arbitres ont-ils juridiction pour décider des moyens de droit présentés au cours de larbitrage par lune des parties?

Jean Gagné

\section{Collaborateurs}

Barrette, Honorable Antonio, D.Sc.S. (h.c.), M.A.L. ministre du Travail de la province de Québec.

Clément, Marcel, Licencié ès lettres, diplômé d'études supérieures de philosophie (Sorbonne), licencié en droit, diplômé d'études supérieures d'économie politique (Faculté de droit de Paris), professeur à la Faculté des sciences sociales de Laval

GAGNÉ, Jean, LL.L., M.Sc.S., avocat, professeur, secrétaire du Département des relations industrielles, Faculté des sciences sociales de Laval.

Tremblay, Gérard, L.Ph., sous-ministre du Travail de la province de Québec, professeur, directeur du Département des relations industrielles, Faculté des sciences sociales de Laval.

Autorisé comme envoi postal de deuxième classe, ministere des postes, Ottawa, Canada.

\section{DE L'ENTREPRISE LIBERALE $\dot{A}$ LA LIBRE ENTREPRISE}

\section{Marcel Clément}

Dans la vie économique comme dans la vie politique, de mauvaises définitions sont souvent génératrices d'équivoque. Et l'équivoque, bientôt entraîne des embarras. Il est en effet, des mots ambigus derrière lesquels se dissimulent le bon et le mauvais, au plus grand dommage de ceux qui les emploient. C'est à quoi nous avons pensé bien souvent, en écoutant certaines apologies de la libre entreprise, derrière lesquelles se cachent mal des tentatives, en vue de réveiller dans les esprits, le culte de ce que Pie XI appelait "les idoles du libéralisme »"

Il est donc, à l'heure où l'édification d'un ordre social catholique s'avère pour les sociétés une question de vie ou de mort, plus important que jamais de préciser les idées sur ce point et de dissiper les malentendus. En effet, si ia libre entreprise, celle qui défend l'initiative personnelle contre les empiètements de l'état est un idéal légitime, l'entreprise libérale, celle qui a institué la loi de la jungle dans l'économie et avili le travail au point d'en faire une marchandise, doit disparaître.$$
-\mathrm{I}-
$$ \\ L'entreprise libérale}

L'entreprise libérale est celle où tout est organisé en vue de faire de la concurrence le mécanisme essentiel de la vie économique. Cette concurrence s'exerce à la vente, sur le marché des produits, comme à l'achat, sur le marché du travail. Les conséquences d'un semblable régime sont le déséquilibre économique et la dissolution sociale. Nous n'envisagerons ici que le cas du marché du travail.

Dans le régime libéral pur, les travailleurs sont isolés. Selon la rareté ou l'abondance de la main-d'oeuvre, l'entrepreneur peut embaucher à un salaire plus ou moins élevé. On a vu dans la première moitié du $19 \mathrm{e}$ siècle, à quels excès un semblable système a pu aboutir. Les besoins du travailleur et de sa famille sont systématiquement oubliés. Plus la main-d'oeuvre sera abondante et par conséquent plus sera considérable l'offre de travail et parallèlement, plus les salaires tendront à s'avilir.

(1) Pie XI "Quadragesimo Anno" in "La Communauté humaine" No 478. 
Le système était trop inhumain pour pouvoir durer. En dépit des interdictions légales, les ouvriers parvinrent à s'unir afin d'opposer à la force patronale une force susceptible de la contrebalancer. C'est ainsi qu'est né le régime néolibéral, celui dans lequel nous vivons.

Dans une telle structure, le régime de la concurrence joue encore sur le marché des denrées (sauf cas de monopole) mais non plus sur le marché du travail où il se trouve limité.

Dans la négociation d'une convention collective, capital et travail sont face à face et se livrent à une épreuve de force. Le salaire dans une pareille structure ne résulte plus, comme dans le régime libéral pur, de la concurrence entre les travailleưrs. Il résulte d'une confrontation, de caractère agressif, entre les besoins familiaux et sociaux invoquées par le monde du travail d'une part, et la prospérité économique de l'entreprise et la juste rémunération des entrepreneurs invoquées par le monde du capital, d'autre part.

Dans un tel système en effet, les salariés sont en permanence appelés à revendiquer. Tout se passe, dans notre société, comme si le salarié avait deux obligations complémentaires: $1^{\circ}$ travailler; $2^{0}$ justifier le taux de son travail. Il est difficile d'imaginer condition plus humiliante.

Les conséquences psychologiques et sociales d'une telle structure économique sont bien connues. Alors que dans l'entreprise, les apporteurs de travail et les apporteurs de capitaux concourent à une oeuvre commune: la prospérité de l'entreprise, la loi de la jungle économique veut qu'ils ne cessent à aucun moment de se regarder comme des rivaux.

Les patrons d'une part s'inquiètent de la reprise de conscience qu'il décèle dans le monde du travail. D'aucuns se demandent si le patron est encore maìtre dans sa maison. Imprégnés, à leur insu, d'esprit libéral, ils estiment que si l'entreprise appartient au propriétaire, il en découle qu'il a, seul, le droit de la diriger. Ils constatent que ce droit est limité par les unions ouvrières par la convention collective, par l'Etat. Et ils voudraient revenir en arrière et redevenir «maîtres dans leurs maisons », sans voir que c'est de l'échec douloureux de cette «maîtise 》 là que sont nées les limitations actuelles.

L'ouvrier, d'autre part, souffre profondément de n'être dans l'entreprise qu'un vendeur de travail et de ne pas se sentir associé matériellement et moralement à l'oeuvre commune qu'exprime si bien la notion de communauté d'entreprise. Il souffre d'être toujours contraint d'avoir à souligner ses besoins pour recevoir un juste salaire, ce qui fait de lui un mineur sur le plan économique, s'il se compare à la situation du propriétaire d'entreprise ou du commerçant dont les revenus dépendent de l'habileté technique et non de la revendication humaine. Il souffre, enfin, de sentir que sa renvendication, même lorsqu'elle est manifestement légitime, est toujours considérée par le patron comme un événement fâcheux et l'intervention des syndicats et des unions comme une limitation de sa liberté de patron.

Ainsi, il serait difficile d'inventer un régime économique susceptible de provoquer aussi mécaniquement et avec une telle rigueur, la discorde sociale.

$$
\text { - II - }
$$

\section{L'entreprise dirigée}

On oppose habituellement le dirigisme au libéralisme, et dans une certaine mesure cette opposition est légitime. A l'initiative privée des entrepreneurs sont substituées les décisions de l'Etat et c'est ainsi qu'est née l'idée de l'entreprise dirigée ce qui signifie exactement «dirigée par l'Etat». Toutefois on n'a peut-être pas assez souligné, jusqu'ici, que l'entreprise dirigée n'avait vu le jour que comme la fille légitime de l'entreprise libérale. Autrement dit l'histoire des cent cinquante dernières années montre clairement que c'est le libre jeu du libéralisme dont le fruit nécessaire n'est autre que l'étatisme. Il n'est pas mauvais d'examiner comment s'opère une telle évolution.

En premier lieu, le libéralisme incite les travailleurs à réagir. Ils renoncent à se concurrencer et s'organisent en union. On a vu au 19e siècle, en Europe, ces unions servir de bouillon de culture au socialisme. Par conséquent, c'est par leur impuissance en face du patronat au sein de l'entreprise libérale que les ouvriers ont été amenés d s'enthousiasmer à l'idée d'une prise en charge par l'Etat de toute la vie économique. Si l'on se réfère au syndicalisme français, on constate en effet, que la tendance réformiste aussi bien que la tendance révolutionnaire correspondent à des moyens différents, dirigés vers un but unique: la conquête du pouvoir politique par la classe ouvrière et au-delà la dictature économique par cette même classe de toutes les entreprises de la nation, 
revanche collective, par des moyens politiques, sur le patronat comme tel.

Si l'on regarde l'histoire de l'Europe au cours des cinquante dernières années, on constatera que c'est là une évolution commune à tous les Etats. Or, de cette évolution, on connaît les résultats !

Au fur et à mesure que, dans un Etat, l'économie est prise en charge par des fonctionnaires, on entre dans le cercle infernal d'une accumulation de réglementations qui étrangle les initiatives les plus légitimes et d'une incompétence administrative qui décourage toute espèce d'effort de production sérieux. Le patron est réduit à un rouage fonctionnarisé qui se voit progressivement imposé, par des étrangers à la profession, une politique des prix, une politique des salaires, parfois même une politique des fabrications . . .

Si ce dirigisme échoue, l'état n'a plus même le recours de jouer son rôle d'arbitre entre patrons et ouvriers puisqu'il est devenu lui-même un état patron. C'est ce qui se passe aujourd'hui en France dans certains secteurs nationalisés. (Houillières).

Dans un pareil système, on sait ce que devient la psychologie patronale. Elle évolue progressivement vers une complète indifférence, sinon avec le dégoût et la révolte. Quant aux salariés, ils se trouvent eux-mêmes victimes de cette dissolution de l'esprit patronal. Ils n'ont plus aucun recours contre le niveau de vie anormalement bas auquel les contraint une mauvaise gestion venant de l'état lui-même. Il est significatif, en effet, de constater que partout où l'état a pris en charge la vie économique, la condition ouvrière a été particulièrement dure et la pauvreté générale surmontée par une propagande qui promet l'âge d'or pour la génération suivante (Cas de trois états prolétariens: le communisme russe, le nationalsocialisme allemand, le fascisme socialiste italien.)

En définitive, le bilan des divers dirigismes s'est exprimé par la ruine économique des pays où ils ont été instaurés, par la disparition de l'esprit d'entreprise et par la caporalisation des travailleurs, trompés par ceux-là mêmes qui gouvernaient le pays en leur nom.

Ce triste destin de l'entreprise dirigée est bien connu. Ce qui l'est moins, c'est le fait que c'est la malfaisance interne du régime libéral luimême qui a régulièrement engendré les utopies dirigistes et que, par conséquent, on ne saurait trop mettre en garde le patronat canadien à ce propos. Ceux qui font aujourd'hui une propagande tenace pour la libre entreprise parce qu'ils redoutent légitimement de voir peu à peu naître l'entreprise dirigée doivent prendre garde de ne pas chercher plus ou moins inconsciemment à renforcer, au nom de la libertà de l'entreprise le libéralisme des conditions du travail. Car il y a des lois en sciences sociales, et l'on peut être sûr que plus les ouvriers verront le patronat accentuer un retour au libéralisme et plus ils tourneront leurs regards vers l'état. Ainsi, il est possible de formuler clairement la situation. Renforcer le libéralisme dans l'entreprise, c'est préparer le dirigisme à longue échéance. Instituer la libre entreprise, c'est rejeter du même coup libéralisme et dirigisme parce que c'est instituer l'esprit chrétien au sein de la communauté de travail.

$$
\text { - III - }
$$

\section{La libre entreprise}

L'économie libérale n'est pas une économie de liberté, c'est une économie de licence. L'économie dirigée n'est pas une économie rationnelle, c'est une économie de contrainte. La libre entreprise doit donc se définir par opposition à l'entreprise libérale en même temps que par opposition à l'entreprise dirigée, car la liberté véritable se définit autant en se distinguant de la licence qu'en se distinguant de la contrainte.

Dans la mesure où elle s'oppose à l'entreprise libérale, la libre entreprise réalise les conditions de la liberté de tous ceux qui participent à l'oeuvre commune.

Elle doit être définie d'abord du point de vue patronal. Il va de soi que l'action syndicale et la négociation des conventions collectives, ne sont pas là du tout pour limiter la liberté patronale, mais seulement pour prévenir les licences. Pour le montrer, il faut distinguer avec soin, le rôle social et le rôle économique du patron. Sur le plan social le patron est ie chef d'une communauté humaine. Il a la charge des conditions de la vie physique, morale et familiale de ses salariés, dans la mesure où ces conditions dépendent de lui. En tant que tel, sa liberté n'a donc d'autre portée que celle du libre arbitre; il est libre de remplir ou de ne pas remplir les devoirs sociaux qui lui incombent. Sur le plan économique, au contraire, le patron jouit d'une véritable indépendance. II détermine les fins économiques de l'entreprise, il met en oeuvre la politique économique susceptible de les réaliser. Surtout il est l'âme de la communauté de travail décidant non seulement de la répartition des tâches, mais insufflant en outre l'enthousiasme à chacun et à tous. Toutefois, un 
problème se pose: ce ne sont pas des machines que le patron dirige dans l'entreprise mais des hommes, des hommes qui sentent et qui jugent. La liberté patronale ne consistera donc jamais à mécaniser totalement la vie professionnelle de ses salariés. La libre entreprise, en effet, doit remplir les conditions de la libération humaine optima de tous ceux qui la constituent. C'est donc seulement dans la mesure où le patron, «tempérant quelque peu le contrat de travail par des éléments empruntés au contrat d'association », réussira à faire de ses salariés de véritables associés, tant sur le plan matériel (réformes de structure) que sur le plan moral (le patron premier ouvrier de l'entreprise), que celle-ci réalisera vraiment une authentique communauté sociale catholique et non plus seulement une transaction commerciale sur du travail humain.

Il faut aussi définir, en effet, la libre entreprise du point de vue du salarié. Un homme n'est pas libre lorsqu'il n'est pas libéré du souci permanent de pouvoir vivre décemment ainsi que sa famille. Un homme n'est pas libre qui ne peut pas se réunir avec ses compagnons de travail en vue d'examiner les problèmes communs. Ces deux libertés essentielles étaient refusées par la loi aux travailleurs dans l'entreprise libérale pure. Elles correspondaient à la possibilité d'une véritable licence du côté patronal: licence de verser des salaires inférieurs au minimum vital, licence d'user de la loi pour empêcher les unions ouvrières. Sans doute le patron n'était pas contraint d'agir ainsi mais le régime de concurrence est une invitation pressante et qui ressemble étrangement à une tentation. Aujourd'hui encore et bien que la loi favorise dans un esprit chrétien le progrès social il existe des entreprises néo-libérales où l'action syndicale est nulle, où les conventions collectives n'existent pas.

Hâter l'évolution de ces entreprises représenterait donc un pas authentique vers la libre entreprise définie comme le lieu de l'épanouissement de toutes les personnes humaines qui $y$ travaillent.
D'autre part, en tant qu'elle s'oppose à l'entreprise dirigée la libre entreprise ne trouvera les vraies conditions de sa libération que dans l'organisation professionnelle. En effet, de même que le meilleur moyen pour éviter la fixation arbitraire des salaires par l'Etat est de laisser les parties intéressées négocier entre elles des conventions collectives, de même il ne sera possible de prévenir la prise en charge étatique de l'économie que par le développement de corps professionnels organisés établissant eux-mêmes la politique générale de la profession. L'entreprise libérale engendre une concurrence qui met les entreprises les plus faibles à la merci des plus fortes. L'entreprise dirigée stérilise l'initiative des chefs économiques réels en la confiant à des fonctionnaires incompétents. La libre entreprise ne pourra s'épanouir qu'au sein d'une organisation professionnelle où les membres de chaque profession composeront ce qui les oppose pour adopter une attitude commune dans l'intérêt commun de la profession.

Pour n'être pas dirigées par l'Etat, cet étranger, les entreprises ne pourront se diriger elles-mêmes librement, étant donnée l'interdépendance croissante de l'économie moderne, qu'à la condition de se diriger en commun, pour pratiquer ensemble de justes prix et de justes salaires.

Au terme de cette analyse, il est possible de dégager l'essentiel de l'esprit chrétien en face des problèmes économiques et sociaux. Le libéralisme a fait de la lutte entre forces inégales le rythme essentiel de toute vie économique. Le dirigisme a fait de la contrainte étatique la technique fondamentale de la vie économique. L'ordre social chrétien charge les intéressés eux-mêmes, de composer, les yeux dans les yeux, et dans un esprit fraternel, les intérêts qui naturellement les opposent les uns aux autres. Des trois formules, il est clair que seule, la dernière, respecte la liberté de l'homme.

\section{Réformes de Structure dans l'Entreprise}

Reproduction des articles publiés dans le Bulletin des relations industrielles par MM. P.-E. Bolté, Marcel Clément et Gérard Dion. Brochure d'environ 150 pages qui sera mise en vente prochainement par le Département des relations industrielles. 